\title{
Classification of CYP450 1A2 inhibitors using PubChem data
}

\author{
Sergii Novotarskyi", lurii Sushko, R Körner, AK Pandey, Igor Tetko \\ From 5th German Conference on Cheminformatics: 23. CIC-Workshop \\ Goslar, Germany. 8-10 November 2009
}

Cytochromes P450 (CYP450) are a superfamily of enzymes, involved in metabolism of a large number of xenobiotic compounds. CYP450 are involved in degradation of a large amount of drugs, currently present on the market. The promiscuity with respect to substrates makes the CYP450 enzymes prone to inhibition by a large amount of drugs, which gives way to clinically significant drug-drug interactions.

In this work different machine learning methods were applied to classify the inhibitors/noninhibitors of human CYP450 1A2. The structures and the active/inactive classification concerning CYP1A2 inhibition were taken from PubChem BioAssay database. This assay uses human CYP1A2 to measure the demethylation of luciferin 6' methyl ether (Luciferin-ME; Promega-Glo) to luciferin.

The tested methods include $\mathrm{k}$ nearest neighbors $(\mathrm{kNN})$, decision tree, random forest, support vector machine (SVM) and associative neural networks (ASNN). The descriptors used were those from the Dragon software, the fragment descriptors and the E-state indices.

The training and test sets were handled separately to avoid different possibilities of overfitting - including overfitting by descriptor selection. Different applicability domain (AD) approaches were used to estimate the confidence of classification.

As a result the models managed to correctly classify $80 \%$ of the test set instances. The accuracy of classification was found to be up to $95 \%$, if only $30 \%$ most confident predictions were taken into account. The model was also applied to an external test set of 187 molecules, collected from literature and measured using a different etalon reaction. For this set accuracy of $78 \%$ was achieved on the $30 \%$ most confident predictions.

Helmholtz Zentrum München, Ingolstädter Landstraße 1, D-85764 Neuherberg, Germany
All the developed models are fast enough to be used for virtual screening of CYP1A2 inhibitors and noninhibitors. The developed models are publicly available on-line at the http://qspr.eu web site.

Published: 4 May 2010

doi:10.1186/1758-2946-2-S1-P40

Cite this article as: Novotarskyi et al:: Classification of CYP450 1A2 inhibitors using PubChem data. Journal of Cheminformatics 2010 2(Suppl 1):P40.

\footnotetext{
Publish with ChemistryCentral and every scientist can read your work free of charge

"Open access provides opportunities to our colleagues in other parts of the globe, by allowing anyone to view the content free of charge." W. Jeffery Hurst, The Hershey Company.

- available free of charge to the entire scientific community

- peer reviewed and published immediately upon acceptance

- cited in PubMed and archived on PubMed Central

- yours - you keep the copyright

Submit your manuscript here:

http://www.chemistrycentral.com/manuscript/

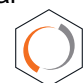
ChemistryCentral
} 